\title{
Utilización de inmunoreacciones para la detección de Scrapie en Óbex de ovinos provenientes de la XII Región de Chile
}

\author{
GUSTAVO FARÍAS F. ${ }^{1}$, M.V., M.SC., ALVARO MACHUCA A. ${ }^{1}$, M.V., CRISTIÁN BRAVO V. ${ }^{1}$, M.V., MARÍA \\ CRISTINA MADARIAGA J. ${ }^{1}$, M.V., CECILIA JARA O. ${ }^{2}$, M.V. y CLAUDIO LECOCQ P. ${ }^{2}$, M.V.
}

1 Facultad de Ciencias Veterinarias y Pecuarias. Departamento de Patología Animal. Universidad de Chile.

2 Servicio Agrícola y Ganadero de Chile (SAG). Unidad de Patología Pecuaria.

\begin{abstract}
USE OF IMMUNOREACTION FOR DETECTION OF SCRAPIE IN OVINE OBEX FROM XII REGION OF CHILE

Scrapie is an neurodegenerative and infectious disease that affects sheep and goats. The purpose of this study is to compare both tecniques, ELISA and IHC, to determine the application factibility giving the conditions of the laboratory in the country. To develop this assay, fourty obex samples were taken from sheep of the XII Region of Chile and were processed for both techniques ELISA and IHC. The results showed $100 \%$ of the samples negative for Scrapie, using both diagnostic methods. Based in obtained and previously reported results, ELISA technique can be recommended to use in massive screening, since yields reliable and rapid test results and IHC is a confirmatory technique. Both techniques are necessary in the creation of current epidemiological surveillance programs in Chile. ELISA and IHC are easily performed given the current laboratories conditions of the Chilean country.
\end{abstract}

Key words: Scrapie, obex, ELISA, IHC.

\section{RESUMEN}

El Scrapie es una enfermedad infecciosa neurodegenerativa que afecta a ovinos y caprinos. Dada la importancia que tiene esta enfermedad es que se desarrolló este estudio enmarcado en la comparación de la técnica de ELISA y la de inmunohistoquímica en el diagnóstico de Scrapie, para determinar la factibilidad de aplicarlas en las condiciones de laboratorio país. Para ello se procesaron 40 muestras de óbex de ovinos en duplicado, provenientes de la XII Región de Chile, las cuales fueron sometidas paralelamente a la técnica de ELISA e IHQ. Como resultados se obtuvo un 100\% de

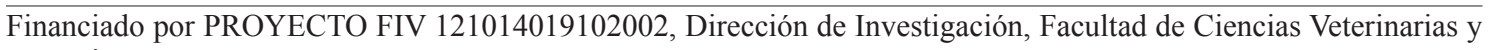
Pecuarias.

Casilla 2, Correo 15, Santiago, Chile.

E-mail: gfarias@uchile.cl 
muestras negativas a Scrapie, sometidas a ambos métodos diagnósticos. Con los valores informados previamente y los obtenidos en esta investigación, es posible recomendar el ELISA como una técnica utilizable en muestreos masivos, ya que aporta resultados confiables de manera más rápida. La IHQ corresponde a una técnica confirmatoria y que ambas, son necesarias en la creación de programas de vigilancia epidemiológica activa en Chile, siendo factibles de realizar en las actuales condiciones de laboratorio país.

Palabras clave: Scrapie, óbex, ELISA, IHQ.

\section{INTRODUCCIÓN}

El Scrapie, enfermedad conocida hace más de 250 años, que afecta tanto a ovinos como caprinos, es el prototipo de un conjunto de enfermedades progresivas neurodegenerativas llamadas Encefalopatías Espongiformes Transmisibles (EETs), que afectan a los mamíferos (Detwiler y Baylis, 2003).

El agente causal de estas enfermedades es una proteína infecciosa que recibe el nombre de prión (PrPSc), la que tiene su origen en una proteína constitutiva de la membrana celular (PrPC) que cambia su estructura conformacional post traduccional, convirtiéndose en la proteína anómala (PrPSc) (Prusiner, 1998).

El Scrapie presenta largos períodos de incubación, signos clínicos como prurito intenso y pérdida de la condición corporal, que se producen sólo poco tiempo antes de la muerte del animal, lo que dificulta su diagnóstico, aumentando así la posibilidad de transmisión y diseminación de la enfermedad a otros animales de especies susceptibles (Van Keulen et al., 2008). Su importancia radica no sólo en las grandes pérdidas económicas que produce en los rebaños afectados, sino que también y más importante aún es la comprobación, en el año 1986, del salto interespecie que es capaz de realizar el agente causal del Scrapie. Es así como, bovinos alimentados con harinas de carne y hueso $(\mathrm{HCH})$ contaminadas con este prión, reprodujeron la enfermedad, pero con algunas variaciones, dando origen a la Encefalopatía Espongiforme Bovina (EEB) (Smith y Bradley, 2003). Esto trajo consigo un mayor impacto, al confirmarse el paso del prion de la EEB al humano, el que adquiere el agente luego de consumir productos y subproductos de bovinos infectados, manifestando clínicamente una variante de la enfermedad de Creutzfeldt-Jakob (vECJ) (Will, 2002; Ducrot et al., 2008). Si bien la transmisión del Scrapie hacia el humano es un hecho no confirmado, tampoco se ha podido descartar esta posibilidad.

Respecto a las EETs, Chile se encuentra actualmente en etapa de demostración del estado zoosanitario frente a la OIE. Esta situación se orienta principalmente a la EEB, hecho que preocupa de sobremanera, ya que se subestima la importancia epidemiológica del Scrapie y otras EETs, junto a sus consecuencias económicas, lo que se observa en la práctica, en las escasas medidas de vigilancia epidemiológica específicas y más rigurosas para el Scrapie.

Con el objetivo de buscar alternativas para ayudar a la solución de este problema, se desarrolló esta investigación, comparando dos técnicas reconocidas internacionalmente para diagnosticar Scrapie, la inmunohistoquímica (IHQ) y el inmunoensayo enzimático (ELISA), en las condiciones de laboratorio país.

Las bases inmunológicas de ambas técnicas son semejantes, es decir, la detección de la presencia de PrPSc asociada a la enfermedad (Machuca et al., 2006; Farías et al., 2007), no obstante, poseen diferencias importantes, las que fueron analizadas, con el propósito no sólo de aportar conocimientos respecto al diagnóstico de la enfermedad, sino también con el objetivo de buscar métodos diagnósticos aplicables a la realidad chilena.

\section{MATERIALES Y MÉTODOS}

Se analizaron 40 muestras de óbex de ovinos, mayores de 2 años de edad, provenientes de mataderos de la XII Región de Chile, sin importar raza ni sexo. Cada muestra en duplicado fue sometida a las 2 pruebas diagnósticas: inmunoensayo enzimático (ELISA) e inmunohistoquímica (IHQ). 


\section{Inmunoensayo enzimático (ELISA)}

Para la detección de proteínas priónicas (PrPsc) mediante esta técnica, se utilizó un kit comercial (TeSeE, Bio-Rad), el que consta de dos partes, la primera de purificación y concentración de proteínas priónicas de las muestras y la segunda de detección mediante inmunoensayo de captura en microplaca (ELISA).

1) Purificación: de cada muestra se obtuvieron $350 \mathrm{mg}$ de óbex, los que fueron sometidos a 2 ciclos de 45 seg de trituración con agitación. Entre ambos se realizó una centrifugación a 10.000 x g por $1 \mathrm{~min}$. Luego las muestras fueron calibradas en duplicado, obteniendo $250 \mu \mathrm{l}$ de la solución de cada triturado, a las que se les agregó 250 $\mu \mathrm{l}$ de una solución de proteinasa $\mathrm{K}(20 \mu \mathrm{l} / \mathrm{ml})$, éstas fueron homogeneizadas e incubadas por 10 min a $37^{\circ} \mathrm{C}$. Posteriormente se adicionaron 250 $\mu l$ de una solución de precipitación más azul de bromofenol. Para concentrar la proteína priónica patógena, las soluciones fueron centrifugadas a $12.000 \mathrm{x}$ g por $7 \mathrm{~min}$ a $20^{\circ} \mathrm{C}$. Se eliminó el sobrenadante y se agregó a cada tubo $25 \mu \mathrm{l}$ de un tampón de solubilización, incubándose por $5 \mathrm{~min}$ a $100^{\circ} \mathrm{C}$. Finalizada esta etapa, se obtuvieron soluciones uniformes de cada una de las muestras de estudio, con las que se llevó a cabo la detección de PrPSc por ELISA.

2) Detección: se utilizaron 80 pocillos de la microplaca para las 40 muestras previamente purificadas y su duplicado. Cada pocillo estaba recubierto con un anticuerpo monoclonal antiPrP. Además, se preparon los controles, el positivo con un péptido sintético no infeccioso en tampón PBS (137 mM NaCl; 2,7 mM KCl; $8,1 \mathrm{mM} \mathrm{Na} \mathrm{HPO}_{4} ; 1,5 \mathrm{mM} \mathrm{KH} \mathrm{PO}_{4} ; \mathrm{pH} \mathrm{7,4)} \mathrm{y}$ el negativo en base a PBS pH 7,4 suplementado con albúmina sérica bovina (BSA). Todas las muestras purificadas se diluyeron agregando a cada tubo $125 \mu \mathrm{l}$ de tampón PBS pH 7,4, suplementado con BSA y rojo fenol.

En la microplaca se distribuyeron en los pocillos, $100 \mu 1$ de cada muestra, así como también de los controles positivos y negativos, los que fueron incubados por $75 \mathrm{~min}$ a $37^{\circ} \mathrm{C}$. Se lavaron y se agregaron $100 \mu \mathrm{l}$ de una solución de conjugado, que contiene el segundo anticuerpo monoclonal anti-PrP conjugado con peroxidasa y se incubó por $60 \mathrm{~min}$ a $4^{\circ} \mathrm{C}$, seguida por 5 ciclos de lavado, para luego agregar $100 \mu \mathrm{l}$ a cada pocillo de una solución de revelado (ácido cítrico más acetato de sodio $\mathrm{pH} 7,4, \mathrm{H} 2 \mathrm{O} 2$ al 0,015\% y dimetilsulfóxido 4\% más el cromógeno Tetrametilbenzidina). La inmunoreacción se detectó en un lector de ELISA $(\kappa=450 \mathrm{~nm})$ a través de la medición de su densidad óptica (D.O.) (Bio-Rad, 2003). En base a los valores promedios de D.O., se validó la placa y se analizaron e interpretaron los resultados de las muestras, calificándolas como positivas, negativas o sospechosas según lo descrito por Bio-Rad (2003) y reproducido por Padilla (2004).

Las muestras fueron analizadas de acuerdo al valor umbral (V.U.) calculado (Bio-Rad, 2003). Así las positivas fueron mayor o igual al V.U., las sospechosas correspondieron a las que se encontraron justo debajo del V.U., también llamado valor límite (V.U - 10\%), y las negativas las menores a dicho valor.

\section{Inmunohistoquímica}

Las muestras de óbex en duplicado fueron inmersas en formalina neutra al $10 \%$ para su fijación. Pasado este tiempo se laminaron, procesaron y cortaron, tal como lo describe Machuca (2006), para realizar cortes histológicos de $5 \mu \mathrm{m}$ de grosor.

Tinción histopatológica. Las muestras fueron teñidas con hematoxilina y eosina modificada para EETs tal como los describe Machuca (2006), tinción que permite observar en forma más clara las vacuolas que pueden presentarse en un tejido positivo a Scrapie.

Técnica Inmunohistoquímica: el protocolo de tinción descrito por Machuca et al., (2006), incluyó la inactivación de la actividad endógena de la enzima peroxidasa presente en el tejido mediante la incubación de las muestras en una solución de agua oxigenada al $10 \%$ en metanol absoluto por $10 \mathrm{~min}$. Luego fueron incubadas en ácido fórmico al $98 \%$ por $5 \mathrm{~min}$ a temperatura ambiente y neutralizadas en una solución tampon Tris-HCL 0,1 M, pH 7,6 por 2 min. A continuación las muestras inmersas en una solución recuperadora de antígenos se autoclavaron por $20 \mathrm{~min}$ a $120^{\circ} \mathrm{C}$. Para realizar la inmunotinción se siguió el protocolo del kit Pullman Monoclonal F99/97.6.1 (VRMD, Inc., 2004), descrito por Machuca et al., (2006). Así las muestras se incubaron con proteinasa $\mathrm{K}$ por 90 seg a temperatura ambiente, luego fueron incubadas con el anticuerpo primario monoclonal F99/97.6.1 (Spraker et al., 2002) en una dilución $1: 1.000$ durante $15 \mathrm{~min}$ a $37^{\circ} \mathrm{C}$. Posterior a los 
lavados, fueron incubadas con el anticuerpo secundario policlonal anti-ratón biotinilado y conjugado con streptavidina-HRP, finalizando con el revelado de la reacción inmunológica mediante la incubación con sustrato de cromógeno AEC (3-amino-9-ethilcarbazol) por 20 min. Por último, se tiñó el parénquima con una solución de contraste (hematoxilina de Mayer) por $10 \mathrm{~min}$.

Para el análisis descriptivo de las muestras, se usaron controles de óbex ovino positivos y negativos aportados por organismos canadienses (Laboratorio de Otawa, Canadian food Inspection Agency), determinando una muestra como positiva ante la presencia de inmunotinción específica en el tejido acompañada de lesiones vacuolares simétricas y bilaterales; como negativa en completa ausencia de inmunotinción, con o sin lesiones vacuolares, y sospechosa ante la presencia de inmunotinción no aclaratoria en zonas específicas del óbex ovino (Machuca, 2006).

\section{RESULTADOS}

ELISA. Para calcular el valor umbral se tomó el promedio de las lecturas (D.O.) de los controles negativos, más una constante 0.210 , resultando un valor de 0,22075. El valor límite

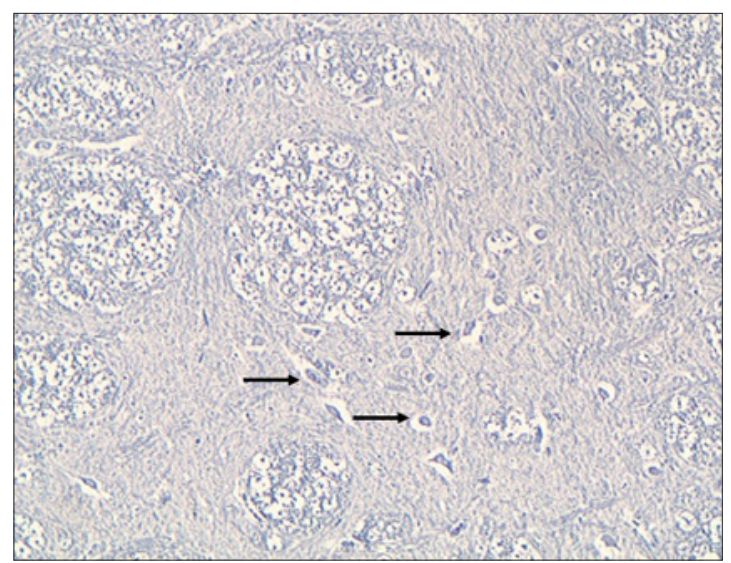

Figura 2. Microfotografía de un corte de óbex ovino sometido a IHQ, correspondiente a un control negativo. Microfotografía de un corte de la médula oblongada a nivel de óbex de ovino, que corresponde a un control negativo, para la tinción IHQ. Con la tinción de contraste con hematoxilina de Mayer, se evidencian los somas neuronales normales (flechas)(200x). calculado (V.U - 10\%) fue de 0,198675 unidades de D.O. En base a estos datos se obtuvo que todos los controles positivos presentaron lecturas mayores o iguales a 1 , y los negativos menores o iguales a 0.0108 , por lo que el total de las 40 muestras de óbex de ovinos y sus duplicados resultaron ser negativos a Scrapie (Tabla 1).

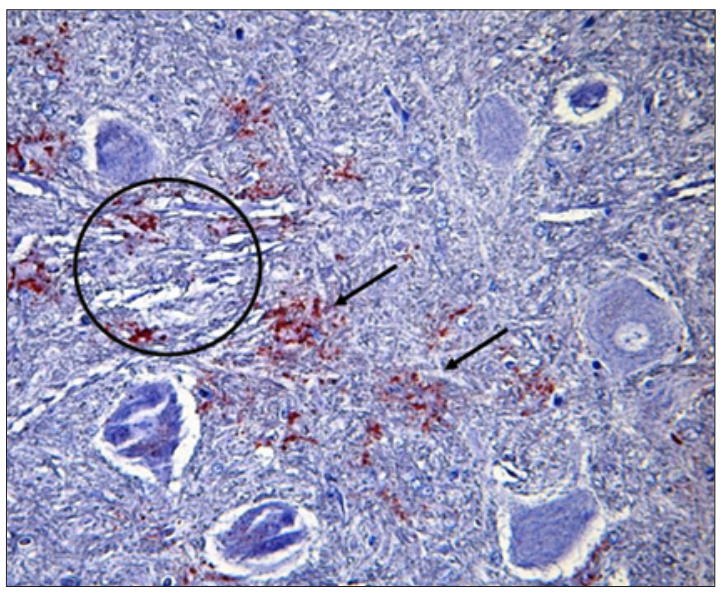

Figura 1. Microfotografía de un corte de óbex ovino sometido a IHQ, correspondiente a un control positivo. Microfotografía de un corte histológico de la médula oblongada a nivel de óbex, tratado según el protocolo de IHQ. Se observan numerosas vacuolas (círculo) y abundante inmunoprecipitado de color rojo (flechas) dado por el cromógeno AEC, el que indica la presencia de PrPSc, (400X).

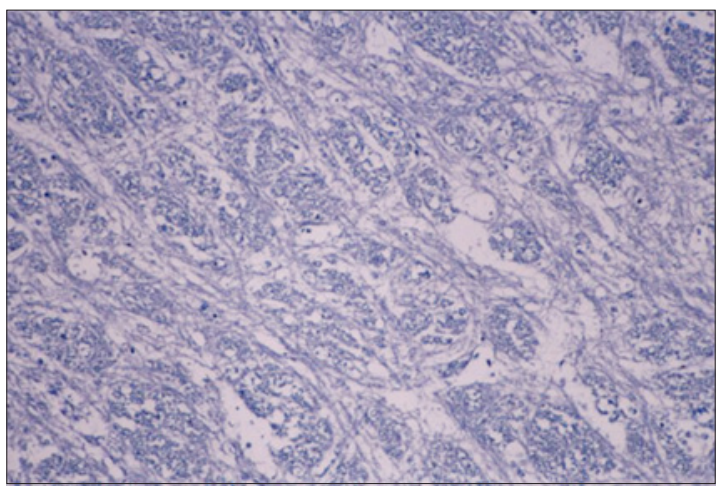

Figura 3. Microfotografía de una de las muestras en estudio sometidas a IHQ. Microfotografía de un corte de médula oblongada a nivel de óbex, de una de las muestras en estudio sometida a la reacción IHQ. El tejido se observa sin inmunoprecipitado de color rojo, lo que indica que esta muestra es negativa a Scrapie según el protocolo de IHQ realizado (100X). 
Tabla 1. Cuadro resumen resultados ELISA

\begin{tabular}{lccr}
\hline & Controles & $\begin{array}{c}\text { Muestras } \\
\text { Analizadas }\end{array}$ & Total \\
\hline Positiva & 2 & 0 & 2 \\
Negativa & 4 & 40 & 44 \\
Sospechosa & 0 & 0 & 0 \\
Total & 6 & 40 & 46 \\
\hline
\end{tabular}

IHQ. Realizada la inmunohistoquímica se observó que la totalidad de los controles positivos presentaron inmunoprecipitado de color rojo, ademas de vacuolas que indican la localización de PrPSc asociada a Scrapie (Figura 1). Por otra parte, en conjunto con los controles negativos (Figura 2), la totalidad de las muestras provenientes de la XII Región de Chile, fueron negativas a la presencia de inmunoprecipitado y de lesiones vacuolares (Figura 3), demostrando que el $100 \%$ de de las muestras analizadas fueron negativas a Scrapie.

\section{DISCUSIÓN}

Históricamente, el diagnóstico inicial de cualquiera de las EETs se basó en la observación de cambios histopatológicos en el tejido nervioso. Con el transcurso del tiempo y el avance en las investigaciones respecto a la patogenia de estas enfermedades, se ha determinado que el principal elemento diagnóstico de búsqueda es la PrPSc asociada a la enfermedad (Gavier-Widén et al., 2005; Parveen et al., 2005), ya que es el único marcador no ambiguo disponible. Las dos técnicas analizadas en este ensayo están basadas en la detección de este marcador.

Los pequeños rumiantes suman, al año 2007, más de 5 millones de cabezas en el país, siendo los ovinos la masa más importante, con casi 4 millones de animales (INE, 2008). Esta gran cantidad de animales, sumado a la relación epidemiológica que posee el Scrapie con la EEB, la posibilidad de que los ovinos se comporten como "vector" de la EEB que produce la vECJ en los humanos y la clara importancia económica que esta enfermedad tiene, al considerar la apertura de nuevos mercados internacionales, son algunas de las razones que justifican la aplicación de un programa de vigilancia epidemiológica para el
Scrapie a nivel nacional. A través del desarrollo y comparación de dos inmunorreacciones para el diagnóstico de Scrapie: IHQ y ELISA, este estudio buscó aportar métodos diagnósticos para su aplicación en un programa de vigilancia país.

LaOIE indica que mientras la técnica de ELISA es un test para screening, es decir, es una prueba de alta sensibilidad diagnóstica, para ser utilizada a gran escala en terreno, la IHQ es confirmatoria, método que posee alta especificidad diagnóstica, por lo que es utilizada para confirmar resultados, generalmente sospechosos o positivos, que se han derivado de la aplicación de otra técnica (OIE, 2000; 2004). Las pruebas de screening deben tener alta sensibilidad para poder detectar a todos los animales infectados, característica que toma más importancia en países que poseen baja prevalencia para una enfermedad específica, como es el caso del Scrapie en Chile, que a la fecha, no ha sido descrito (OIE, 2008).

La IHQ está definida como técnica confirmatoria, esto porque a la observación microscópica no sólo basta observar la presencia de inmunoprecipitado en las zonas específicas del óbex, sino que además se busca que exista un patrón lesional característico para la enfermedad (Parveen et al., 2005). Por estas razones se trataría de la prueba más específica disponible en la actualidad (Gavier-Widén et al., 2005). Estas características son las que obligan a que la interpretación final de los resultados, sea realizada por un Médico Veterinario Patólogo especializado, por lo que no resulta adecuada para ser usada en programas de screening (Parveen et al., 2005). La anterior situación se contrasta con la técnica ELISA ya que, si bien para el desarrollo de este método y la posterior interpretación de los resultados es necesario personal entrenado, no es fundamental que se trate de especialistas en la materia, ya que el entrenamiento resulta mucho más sencillo que para la IHQ y puede ser realizado sólo por una persona entrenada (Farías et al., 2007). Por lo tanto, el kit TeSeE demostró ser más sencillo de realizar que la IHQ.

Respecto a los tiempos de realización de ambas técnicas, también se observaron grandes diferencias. Desde que las muestras ingresan al laboratorio, hasta la entrega de resultados informados, en la técnica de ELISA se necesitaron aproximadamente $6 \mathrm{~h}$, mientras que para la IHQ se precisaron aproximadamente $48 \mathrm{~h}$. Pese a que el protocolo de IHQ utilizado demoró menos 
tiempo que otros revisados en la bibliografía, el tiempo utilizado es ostensiblemente mayor al de ELISA. Así, para la realización de screening masivos, es fundamental utilizar técnicas que entreguen resultados de manera rápida, por lo tanto, se observa que sólo el ELISA sería apropiado para estos fines.

Luego de realizar el ELISA se observó que el porcentaje de especificidad obtenido a través de esta técnica fue de un $100 \%$, tal como lo obtenido por Padilla (2004) y lo aportado por la literatura (EC, 1999; VLA, 2004; EFSA, 2005). Misma situación ocurrió con la IHQ, la cual presentó también $100 \%$ de especificidad, lo que coincide con otros estudios realizados (Manning et al., 2001; Machuca, 2006). Sin embargo, no fue posible determinar la sensibilidad para ambas técnicas, debido a que ninguna de las muestras sometidas a este estudio resultó positiva, con excepción de los controles positivos.

Al analizar el tipo de resultados aportados por cada una de las técnicas, podemos observar que los resultados obtenidos a través del ELISA son cuantitativos, lo que favorece su interpretación final, en contraste a lo que ocurre con otras pruebas rápidas, como el Western Blot que arroja resultados cualitativos, los que pueden ser equívocamente interpretados como falsos negativos o falsos positivos (Tabouret et al., 2003), en contraste también a lo que ocurre con la IHQ, resultados que si bien son clasificados con distintos grados de positividad, son cualitativos, ya que la presencia o ausencia de inmunotinción y de lesiones es la que determina el resultado final. Por lo tanto, los resultados aportados por el ELISA son de más fácil interpretación, ya que se trabaja en base a valores numéricos que hacen más difícil la aparición de falsos positivos o negativos, a este nivel del ensayo.

\section{CONCLUSIONES}

Todas las muestras de óbex de ovinos analizadas en este estudio por la técnica de ELISA, resultaron negativas a Scrapie, ya que los valores de D.O. obtenidos fueron inferiores al valor umbral (V.U.) calculado.

Las mismas muestras analizadas por la técnica de IHQ fueron negativas a Scrapie, ya que en ninguna de ellas se observó inmunotinción positiva, así como tampoco lesiones vacuolares en las zonas anatómicas específicas del óbex.

Así, la totalidad de las muestras de óbex de ovinos que fueron analizadas a través de dos técnicas diagnósticas, resultaron negativas a Scrapie.

De acuerdo a los resultados tanto con la técnica de ELISA, como con la de IHQ, se obtuvo un $100 \%$ de especificidad.

La técnica de ELISA corresponde a un método más sencillo que requiere menos tiempo para su realización que la IHQ.

Aunque ambas técnicas son aplicables en el diagnóstico de Scrapie en las condiciones laboratorio país, se recomienda que la de ELISA se utilice para screening y la IHQ para la confirmación de casos sospechosos o positivos, en el marco de un programa de control o vigilancia epidemiológica país.

\section{REFERENCIAS}

1.- BIO-RAD. 2003. Detection des encephalopaties spongiform transmissibles. Test TeSeE. Classeur de formation. Centre de Formation Biologique. Paris, France. $188 \mathrm{pp}$.

2.- DETWILER L, BAYLIS M. 2003. The epidemiology of Scrapie. Rev Sci Tech Off Int Epiz (OIE) 22: 121143.

3.- DUCROT C, ARNOLD M, DE KOEIJER A, HEIM D, CALAVAS D. 2008. Review on the epidemiology and dinamics of BSE epidemics. Vet Res 39: 15-33.

4.- EC (EUROPEAN COMISSION). 1999. Report: The evaluation of tests for the diagnosis of transmissible spongiform encephalopathy in bovines. $37 \mathrm{pp}$.

5.- EFSA (EUROPEAN FOOD SAFETY AUTHORITY). 2005. Evaluation of rapid post-mortem TSE tests intended for small ruminants. EFSA Sci. Rep. 31: 1-17.

6.- FARÍAS G, MACHUCA A, PADILLA D, LECOCQ C. 2007. Detección de proteína prionica de cerebros de bovino mediante inmunoensayo enzimático. Rev Electrón Vet 3(3): 1-7.

7.- GAVIER-WIDÉN D, STACK M, BARON T, BALACHANDRAN A, SIMMONS M. 2005. Diagnosis of transmissible spongiform encephalopathies in animals: a review. J Vet Diagn Invest 17: 509-527.

8.- INE (INSTITUTO NACIONAL DE ESTADÍSTICAS). 2010. Censo agropecuario 2007. Existencia de ganado en las explotaciones agropecuarias y forestales por especie, según región. On line: < http://www.ine.cl/ canales/chile_estadistico/censos_agropecuarios/censo agropecuario_07.php > [Consulta: 24-03-10]. 1 pp.

9.- MACHUCA A. 2006. Detección de Proteína Priónica Patológica ovina mediante inmunohistoquímica en animales provenientes de la XII Región. Memoria Médico Veterinario Facultad de Ciencias Veterinarias y Pecuarias. Universidad de Chile. $81 \mathrm{pp}$.

10.- MACHUCA A,LECOCQ C, JARA C, FARIAS G. 
2006. Detección de proteína priónica patológica ovina mediante inmunohistoquímica en animales provenientes de la XII Región. Monogr Electron Patol Vet 3 (2): 66-81.

11.- MANNING L, ROBINSON Y, CZUB S, WRIGHT P, SPENCER Y, WELLS G, MARSH S, O'ROURKE K, KNOWLES D, ADAMS S. 2001. Validation of an immunohistochermistry assay for the detection of PrPsc in bovine spongiform encephalopathy: an international collaboration. Abstracts from the scientific sessions of the 44th Annual Meeting of the American Association of Veterinary Laboratory Diagnosticians, Hershey, PA. $74 \mathrm{pp}$.

12.- OIE (ORGANIZACIÓN MUNDIAL DE LA SALUD ANIMAL). 2000. Scrapie and BSE En: Manual of standards for diagnostic test and vaccines; $4^{\text {th }}$ edition. $957 \mathrm{pp}$.

13.- OIE (ORGANIZACIÓN MUNDIAL DE LA SALUD ANIMAL). 2004. Manual of diagnostic test and vaccines for Terrestrial Animals. Volumes I and II, $5^{\text {th }}$ Edition. 1178 pp.

14.- OIE (ORGANIZACIÓN MUNDIAL DE LA SALUD ANIMAL). 2010. WAHID Interface. Enfermedades en el tiempo: Prurigo Lumbar. On line: <http://www.oie. int/wahis/public.php?page=home $>$ [consulta: 29-0310]. $6 \mathrm{pp}$.

15.- PADILLA D. 2004. Detección de proteína priónica mediante inmunoensayo enzimático. Memoria Médico Veterinario. Facultad de Ciencias Veterinarias y Pecuarias. Universidad de Chile. 68 pp.

16.- PARVEEN I, MOORBY J, ALLISON G, JACKMAN R. 2005. The use of non-prion biomarkers for the diagnosis of transmissible spongiform encephalopathies in the live animal. Vet Res 36: 665-683.

17.- PRUSINER SB. 1998. Prions. Proc Natl Acad Sci USA 95: 13363-13383.

18.- SMITH P, BRADLEY K. 2003. Bovine spongiform encephalopathy (BSE) and its epidemiology. Br Med Bull 66: 185-198.

19.- SPRAKER T, O'ROURKE K, BALACHANDRAN A, ZINK R, CUMMINGS B, MILLER W, POWERS B. 2002. Validation of monoclonal antibody F99/97.6.1 for immunohistochemical staining of brain and tonsil in mule deer (Odocoileus hemionus) with chronic wasting disease. J Vet Diagn Invest 14: 3-7.

20.- TABOURET G, ANDREOLETTI O, FOUCRAS G, LACROUX C, SCHELCHER F. 2003. Comparaison des tests BioRad TeSeE et TeSeE Petit Ruminants pour la détection des la PrPres, à partir d' èchantillons de tissus lymphoides et de moelle épinière d'ovins à différent stades d'incubation de Tremblante. In: detection des encephalopathies spongiform transmissibles. Test TeSeE. Classeur de formation. Centre de formation biologique. Paris, France. 188 pp.

21.- VAN KEULEN L, BOSSERS A, VAN ZIJDERVELD F. 2008. TSE pathogenesis in cattle and sheep. Vet Res 39: 24-36.

22.- VLA (VETERINARY LABORATORIES AGENCY). 2004. Comparative Testing of three BSE tests: Enfer, Biorad TeSeE, Prionics Check Western. Conducted by the EU Community Reference Laboratory for TSE. 7 pp.

23.- VMRD, INC. 2004. Bovine spongiform encephalopathy antigen test kit, Immunohistochemistry. $8 \mathrm{pp}$.

24.- WILL R. 2002. Variant Creutzfeldt-Jakob disease. Acta Neurobiol Exp 62: 167-173. 